\title{
¿Autoría algorítmica? \\ Consideraciones sobre la autoría de las obras generadas por inteligencia artificial
}

\author{
$* * * *$

\section{Luis Vásquez Leal} \\ Universidad Rafael Belloso Chacín \\ abg.luisvasquez@gmail.com
}

Recibido: 31 de octubre de 2020

Aceptado: 30 de noviembre de 2020

\section{Resumen}

Los avances tecnológicos en materia de inteligencia artificial están haciendo replantear el sistema actual de protección de los derechos de autor. El presente trabajo de investigación busca realizar una aproximación al análisis sobre a quién le corresponde la autoría sobre las obras generadas por inteligencia artificial. Se discute si la legislación actual permite la llamada autoría algorítmica y se evalúan los diferentes escenarios que supone la determinación de la autoría de este tipo de obras. Se debate sobre si la autoría debe recaer en el programador, en el usuario del sistema o en la persona que encarga la obra. Se concluye que actualmente no existe una solución legal expresa y por ende debe procurarse una regulación acorde a los nuevos desafíos tecnológicos con base en el consenso de los diferentes actores que hacen vida en el mundo de la propiedad intelectual.

Palabras clave: autoría, inteligencia artificial, algoritmos, propiedad intelectual.

\section{Algorithmic Authorship? Considerations about the Authorship of the Works Generated by Artificial Intelligence}

\begin{abstract}
Technological advances in artificial intelligence are making rethinking the current system of copyright protection. The present research work seeks to carry out an approach to the analysis on who owns the copyright works generated by artificial intelligence. It is discussed whether current legislation allows the so-called algorithmic authorship and the different scenarios involved in determining authorship are evaluated on this type of works. It is debated whether the authorship
\end{abstract}


rests with the programmer, the system user or the person commissioning the work. It is concluded that there is currently no express legal solution and therefore, a regulation must be sought according to the new technological challenges based on the consensus of the different actors that make life in the world of intellectual property.

Key words: authorship, artificial intelligence, algorithms, intellectual property.

\title{
Autoridade algoritmica? Considerações sobre da autoridade de obras geradas pela inteligência artificial
}

\begin{abstract}
Resumo
Os avanços tecnológicos em inteligência artificial estão fazendo com que o atual sistema de proteção de direitos autorais seja repensado. Este trabalho de pesquisa busca realizar uma abordagem para a análise de quem detém os direitos autorais sobre obras geradas por inteligência artificial. Discute-se se a legislação atual permite a chamada autoria algorítmica e os diferentes cenários envolvidos na determinação da autoria deste tipo de obras são avaliados. É debatido se a autoria deve ficar com o programador, o usuario do sistema ou a pessoa que encomendou o trabalho. Conclui-se que atualmente não há solução jurídica expressa e, portanto, deve-se buscar uma regulamentação de acordo com os novos desafios tecnológicos com base na consenso dos diferentes atores que fazem a vida no mundo da propriedade intelectual.
\end{abstract}

Palavras chave: autoria, inteligência artificial, algoritmos, propriedade intelectual.

\section{Relación entre el derecho de autor e inteligencia artificial}

En la era de los sistemas inteligentes, en los que la ciencia y la tecnología avanzan de una manera vertiginosa para generar nuevas formas de inteligencia artificial (IA), se están generando escenarios complejos y que no se encuentran previstos ni regulados por la legislación actual. El big data, la inteligencia artificial, la robótica, las impresoras $3 \mathrm{D}$ y el internet de las cosas son solo algunas de las tecnologías emergentes que están impactando en los procesos cotidianos de las personas. En ese sentido, ante el desarrollo de la inteligencia artificial, una de las áreas del derecho que se enfrenta a grandes retos es el sistema de la propiedad intelectual, en el que la creatividad computacional y la generación de expresiones artísticas artificiales vienen a desafiar instituciones que hasta hace poco se consideraban sólidas.

Esto hace que surjan grandes interrogantes respecto a los productos de la creatividad artificial y frente a la eventual protección autoral de las llamadas "obras generadas por computadoras". El presente artículo busca realizar una aproximación al análisis de las siguientes preguntas: ¿quién debe asumir la titularidad originaria de los derechos de autor sobre las obras generadas por inteligencia 
artificial? ¿Puede un algoritmo considerarse autor o titular originario a los efectos del sistema actual de la propiedad intelectual? En ese sentido, lo primero que debe hacerse es delimitar lo que se entiende por "inteligencia artificial", a sabiendas de que no existe una definición consensuada de esta.

Si bien en los últimos ańos se han venido realizando grandes avances en ese ámbito, la idea de inteligencia desarrollada por máquinas no es un concepto nuevo. Ya Thomas Hobbes (1989), en su libro Leviatán, planteaba que el hombre, al imitar la naturaleza, tiene la capacidad de crear "animales artificiales", haciendo referencia a los autómatas de la época conformados por artefactos que tenían la capacidad de moverse por sí mismos, como, por ejemplo, el famoso caballero robótico o el león autómata ideados por Leonardo da Vinci. Por su parte, Gottfried Leibniz indicó que los procesos cognitivos del ser humano siguen determinados axiomas de la lógica y que cuando se trata de razonamiento inteligible la mente humana opera conforme a determinados procedimientos algorítmicos implícitos, considerando que la cognición humana puede explicarse en términos computacionales y automatizados (Kulstad y Carlin, 2013).

Por su parte, Alan Turing, científico inglés considerado como uno de los padres de la ciencia de la computación y reconocido por sus valiosos aportes durante la Segunda Guerra Mundial, publicó en el año 1950 en la revista Mind un artículo titulado: "Computing machinery and intelligence", en el que formulaba el siguiente interrogante: ¿pueden las máquinas pensar? Esta pregunta es la base para el método conocido como el Test de Turing, que es utilizado para determinar si una máquina es capaz de desarrollar comportamientos inteligentes similares a los de un ser humano (Frankish y Ramsey, 2014, p. 14).

Pero como tal el término "inteligencia artificial” fue propuesto en el año 1956 cuando John McCarthy lo dio a conocer durante la conferencia de Dartmouth (Dartmouth Summer Research Project on Artificial Intelligence), considerándose como el inicio formal de la inteligencia artificial como área de investigación. Aunque el término "inteligencia artificial" carece de novedad, no ha sido sino en los últimos veinte años que se han desarrollado importantes avances en la materia. En el año 1996, cuando el supercomputador Deep Blue de IBM venció al que para ese entonces era campeón mundial de ajedrez, Gary Kaspárov, se manifestó uno de los grandes hitos en materia de inteligencia artificial.

En el año 2011, el supercomputador Watson, también propiedad de IBM, le ganó a los campeones del reconocido concurso de televisión estadounidense llamado Jeopardy. Más recientemente, en el año 2016, el programa informático AlphaGo, que fue desarrollado por Google DeepMind, le ganó a Lee Sedol, que para ese entonces era el campeón mundial del legendario juego chino Go, que es 
considerado uno de los juegos de estrategia más difíciles del mundo. Lee Sedol describió las jugadas de AlphaGo como estrategias dotadas de una increíble inteligencia y de un alto nivel de creatividad.

Ahora bien, para poder entrar a analizar los retos que la inteligencia artificial significa para la propiedad intelectual, particularmente en relación con la autoría, debe precisarse una delimitación de su definición para los efectos del presente ensayo. Según la Real Academia Española (s.f.), se entiende por "inteligencia artificial" a la "disciplina científica que se ocupa de crear programas informáticos que ejecutan operaciones comparables a las que realiza la mente humana, como el aprendizaje o el razonamiento lógico". En otras palabras, busca lograr la simulación de comportamientos inteligentes en las computadoras.

Pero debe aclararse que cuando actualmente se habla de inteligencia artificial, en realidad se está haciendo referencia al deep learning, que es un área específica de la ciencia de la computación que trata de modelar la actividad que ocurre en la neocorteza cerebral para simular ciertos procesos cognitivos, como el reconocimiento de patrones en contextos digitales como sonidos, imágenes y otros tipos de datos. (Frankish y Ramsey, 2014, p. 15) Tampoco debe confundirse el deep learning con el machine learning. Este último es un campo de la ciencia de la computación que estudia los algoritmos y las técnicas para automatizar soluciones y problemas complejos que son difíciles de programar por medio del uso de métodos convencionales de programación (Gopinath et al., 2019). Es decir, el machine learning utiliza algoritmos que tienen la capacidad de aprender por sí mismos sin que el algoritmo sea programado para obtener un resultado específico. Por su parte el deep learning es una subárea tanto del machine learning como de la inteligencia artificial que utiliza las llamadas "redes neuronales artificiales" para adaptar el algoritmo y que este aprenda de una cantidad masiva de datos.

De manera que el deep learning, o aprendizaje profundo, es una tecnología por medio de la cual se le suministra a la máquina una gran cantidad de datos sobre un determinado dominio para su procesamiento, para que luego el algoritmo pueda efectuar la correspondiente toma de decisiones. Por ejemplo, si se le suministra al algoritmo una cantidad masiva de fotos de gatos, el sistema aprenderá los patrones que los representan y podrá posteriormente identificarlos en una mayor base de datos conformada por imágenes de todo el reino animal sin mayor dificultad. El aprendizaje automático utiliza datos estructurados para perfeccionarse continuamente y tomar decisiones, sin una programación específica, asociadas a la realización de una tarea concreta. Busca este proceso imitar la cognición biológica de una persona. ${ }^{1}$

1 Ver: https://www.wipo.int/about-ip/es/artificial_intelligence/index.html. 
Lo cierto es que actualmente hay una gran cantidad de actividades en las que la inteligencia artificial está superando las habilidades humanas, entre ellas, el procesamiento de macrodatos, el diagnóstico de enfermedades o las predicciones por medio de modelos estadísticos. Como un área aplicada del deep learning y de la inteligencia artificial, ha surgido la creatividad computacional, que se encarga de estudiar y desarrollar programas informáticos capaces de producir obras con un alto contenido creativo. Ejemplos de la creatividad computacional son: el arte visual generativo, la música artificial, la literatura y la poesía generadas por computadora y los artículos generados por sistemas informáticos, entre otros (Boden, 2004).

Para crear expresiones artísticas artificiales, la tecnología del aprendizaje profundo utiliza lo que se denomina "redes neuronales artificiales" para desarrollar determinados procesos creativos. Estas redes neuronales son modelos computacionales que buscan representar a un nivel informático las funciones de las interconexiones que realizan las neuronas del cerebro humano. Lo que hace la creatividad computacional es adoptar una serie de algoritmos, procesos y estructuras para generar nuevas formas de expresión artística. El arte generado por computadora se denomina "arte generativo" y se refiere a cualquier práctica en la que se utiliza un sistema para generar arte de manera artificial. En efecto, el arte generativo se refiere al uso de un sistema usualmente un algoritmo o software con algún grado de autonomía para contribuir en la realización, o propiamente desarrollar, una obra de arte.

Un ejemplo de la aplicación del deep learning es el procesamiento de lenguaje natural, que se encarga de abordar la relación entre el lenguaje humano y las máquinas. Por ejemplo, esta es la misma tecnología que actualmente utilizan los asistentes virtuales como SIRI o Cortana para reconocer los comandos auditivos que son realizadas por el usuario. Una forma de crear expresiones artísticas por medio del deep learning y del lenguaje natural es el uso de algoritmos para la creación de poesía artificial. Un desarrollador llamado Nathan Matias, graduado del Instituto de Tecnología de Massachusetts (MIT, por sus siglas en inglés) puso en marcha un proyecto denominado "Swift-Speare" en el cual se utilizó una base de datos de Shakespeare que, combinada con un algoritmo de escritura de poesía, logró componer un nuevo soneto con un estilo poético similar al de William Shakespeare.

De manera similar, con una cantidad masiva de datos y con el poder de procesamiento computacional de los algoritmos, puede utilizarse el deep learning para crear música, arte visual y diversas formas de expresión creativa. Ahora, en el caso de dichas obras de expresión artística creadas por sistemas de inteligencia artificial, ¿quién debe considerarse como autor de estas? Esta es una de las cuestiones 
más controvertidas en relación con la protección autoral de las obras generadas por computadoras.

\section{La autoría en el sistema actual del derecho de autor}

La calificación de autor corresponde a la persona que crea la obra, de manera que el autor es el sujeto titular originario sobre las obras que crea (Lipszyc, 2017).

De manera que el autor de una obra se entiende como el titular originario de los derechos de esta.

Según el Convenio de Berna, el derecho de autor surge desde el momento de la creación de la obra original, que adquiere protección automática sin necesidad del cumplimiento de formalidades especiales, lo que busca la salvaguarda de los respectivos derechos morales y patrimoniales del autor. En ese sentido, debido a la Doctrina Romántica del Autor que surge durante el siglo XIII, el sistema de derecho de autor actual se basa en el principio personalista de la autoría, según el cual la titularidad originaria de la obra le corresponde en exclusiva a la persona natural que la creó y, salvo ciertas excepciones, se excluye la posibilidad de que la autoría verse sobre entes distintos a una persona natural. En ese sentido, la obra del ingenio se entiende como una prolongación de la personalidad del autor que se exterioriza por medio de su creación.

Aunque el Convenio de Berna no determina el criterio personalista de autoría de una manera expresa, sí lo hace de una manera tácita. Esto se evidencia debido a un conjunto de disposiciones que hacen presuponer la figura del autor humano, como es el caso del artículo 6 que consagra los derechos morales del autor que solo le corresponden a la persona física que crea la obra, o también el supuesto del artículo 7.1, que establece que la protección autoral se extiende durante toda la vida del autor y, como mínimo, cincuenta años después de su muerte (Ramalho, 2017).

En ese orden de ideas, como dice Ricketson (citado en Ginsburg, 2018), aunque el Convenio de Berna no restringe expresamente la autoría como una cualidad exclusiva del ser humano, la doctrina es uniforme al afirmar que, al momento de redactar las disposiciones del convenio, existía un acuerdo tácito entre los Estados partes en relación con el significado de autoría y por eso no era necesario incorporar una definición expresa, considerándose como única interpretación lógica la de considerar como autor a la persona natural que creó la obra. (Ginsburg, 2003). El dilema se basa en analizar si ante el desarrollo tecnológico y el estado de la técnica actual sería necesario adoptar nuevos paradigmas normativos para tutelar la cualidad de autoría de las obras generadas por inteligencia artificial o 
si los principios que establece el Convenio de Berna son suficientes para brindar respuesta a todos los problemas propios del derecho de autor (Sorjamaa, 2016).

Tanto en los países miembros del common law como en los países que forman parte del sistema continental romanista, se entiende que solo una persona natural es quien crea la obra y, por ende, en principio solo la persona natural sería el único titular originario de los derechos de autor sobre ella. El sistema inglés del copyright sigue el modelo del Estatuto de la Reina Ana, que contempla la noción literaria de "autor" como creador (persona natural) que se remonta a la teoría política del individualismo posesivo de Locke, que considera la figura de autor como propietario. Por lo que si el autor es propietario de los derechos intelectuales sobre la obra, entonces solo los sujetos capaces de obligarse legalmente pueden considerarse como autores. Por lo que las máquinas, al carecer de personalidad jurídica, no podrían ostentar la titularidad originaria de las obras del ingenio (Bridy, 2012).

Es por ello que en los Estados Unidos se excluye de manera expresa la autoría por entes no humanos, lo que le impide a los sistemas de inteligencia artificial considerarse como autores. De modo que desde los renombrados Tradermark cases,${ }^{2}$ la Suprema Corte ha dictaminado que la autoría es una cualidad inherente al intelecto de la persona humana. En ese sentido, la originalidad se basa en el "poder creativo de la mente", haciendo referencia a un elemento netamente subjetivo y exclusivo del ser humano. De ahí que, para la Corte, el Copyright es el derecho exclusivo de un sujeto a la protección de lo producido por su propio intelecto (Bridy, 2012).

De manera similar sucede en el ámbito Europeo; el Tribunal de Justicia de la Unión Europea ha declarado en reiteradas oportunidades -como sucede en los casos C-5/08 y C-393/09 - que considera que para que una obra alcance protección autoral, el autor debe expresar sus "decisiones libres y creativas", lo que hace referencia a la figura del autor como persona natural. Por su parte, la Directiva 2009/24/CE 5 sobre la protección jurídica de programas de ordenador consagra en su artículo 2 (1) que se considerará como autor del programa de ordenador a la persona física o al grupo de personas físicas que lo hubieren creado. Aunque se deja a discrecionalidad de cada Estado miembro la posibilidad de conceder la titularidad originaria a una persona jurídica.

2 Tradermark cases 100 U.S. 82 (1879).

3 Tribunal de Justicia de la Unión Europea, Infopaq Asunto C-5/08 REC, P.I-6569 (2009).

4 Tribunal de Justicia de la Unión Europea, Asunto C-393/09 (2010).

5 Artículo 2, Directiva 2009/24/CE del Parlamento Europeo y del Consejo de 23 de abril de 
Pero a diferencia del criterio personalista tácito de la autoría del Convenio de Berna, hay legislaciones que sí contemplan expresamente la figura del autor humano, como la Ley de Propiedad Intelectual Española, ${ }^{6}$ que en su artículo 5.1 establece que se considera como autor a la persona natural que crea una obra literaria, artística o científica. Por su parte, Alemania contempla la figura del autor humano con un criterio absoluto. Con relación a Latinoamérica, también se contempla una concepción personalista del autor; ejemplo de ello es el artículo 3 de la Decisión 351 de la Comunidad Andina de Naciones, que establece el Régimen Común sobre Derecho de Autor y Derechos Conexos y considera como autor a la persona física que realiza la creación intelectual.

Aunque el consenso internacional es considerar a la persona natural como titular originaria de las obras del ingenio, también se consagran excepciones a favor de las personas jurídicas que económicamente han invertido en la creación de la obra. Ese es el caso de la doctrina work made for hire propia de los países del common law, que prevé la titularidad de la cualidad de autoría a favor del empresario respecto a las obras creadas por sus empleados en el ámbito de la actividad para las que han sido contratados (Saiz García, 2019).

También hay otras figuras en las que la autoría es diluida en favor de varias personas que intervienen en el proceso creativo. Tal es el caso de las obras en colaboración en las que intervienen dos o más autores, existiendo una inseparabilidad de las contribuciones individuales de cada uno, por lo que rige la coautoría como ficción legal. La obra colectiva es otra ficción jurídica en la que intervienen varios sujetos en la elaboración de la obra, pero la titularidad originaria es asumida por el colaborador, que es la persona que tiene la iniciativa y dirección de coordinar la realización de la obra (Fernández, 2011).

\section{Creatividad, originalidad y autoría algorítmica}

Existe una relación directa entre la expresión creativa de una obra, su originalidad y la determinación de su autoría. Esto se debe a que la autoría es una consecuencia de la creación intelectual de la obra, por lo que para determinar la autoría necesariamente debe realizarse un análisis del proceso creativo que involucra su creación. De esta manera, para analizar a quién le corresponde la autoría de una determinada obra generada por inteligencia artificial lo primero es determinar cómo se llevó a cabo el proceso de creación de esta. Para ello, debe, a su vez, des-

6 Ley de Propiedad Intelectual 8930, BO 22/04/1996, artículo 5.1. 
pejarse el grado de participación o de influencia de una persona humana durante este. Esto es así porque para que una creación goce de protección autoral, debe tratarse del resultado de una actividad humana (Ruipérez de Azcárate, 2012).

La creatividad es una habilidad que tiende a vincularse con el ser humano y con su intelecto. De manera que, en principio, solo se considera como autor y titular originario del derecho a la persona física que realiza el acto de creación intelectual (Antequera Parilli y Gómez, 1999). Pero lo cierto es que, a lo largo de la historia, han surgido diferentes mitos en relación con la naturaleza de la creatividad, muchos de los cuales aún se mantienen en la cultura popular hasta el punto de influir en las legislaciones y en los principios que conforman el sistema de propiedad intelectual. A este respecto, no es reciente el interés de las personas por comprender cómo se genera la creatividad y de buscar descifrar los misterios que envuelve el proceso creativo. Desde hace siglos, tanto artistas como científicos e investigadores están intentando esclarecer las incógnitas que esta comprende, entender de dónde proviene y qué es lo que hace a las personas ser más creativas.

Ya los griegos y los romanos asociaban la creatividad con la originalidad y consideraban que las obras de pintores y escultores carecían de originalidad, al entender que únicamente imitaban el mundo que veían a su alrededor. Por tales motivos, solo consideraban como originales las obras realizadas por los poetas y músicos porque, según la mitología griega, estos recibían la inspiración de las musas que, como seres espirituales, se pensaba que tenían el don de inspirar el arte. Es gracias a Aristóteles que se comienza a asociar la creatividad con la inteligencia y de considerar el proceso creativo como un atributo del intelecto humano.

Durante la Edad Media, debido a la gran influencia del cristianismo, se considera que la cualidad de ser creativo es una potestad exclusiva de Dios, puesto que solo el ser supremo es el único ser capaz de crear a la imagen y semejanza del ser supremo. Por ende, el hombre no se considera como un ser creativo, sino como un mero instrumento de la divinidad. Esto cambia en el Renacimiento, gracias al humanismo y a la proliferación de las artes y a la renovación de las ciencias, siendo en dicho período cuando el artista se convierte en el verdadero centro de la creatividad.

A finales del siglo XVIII surge la corriente del romanticismo, que considera la creatividad como un privilegio del artista, hasta el punto de llegar a considerarse como un atributo exclusivo y característico de su personalidad. De ahí surge la concepción romanista del autor, mediante la cual se considera la expresión creativa como característica exclusiva del ser humano, lo que todavía influye y se mantiene en las actuales legislaciones de propiedad intelectual. 
Esto se demuestra tanto en la legislación como en la jurisprudencia de los diferentes países. En el caso Bleistein v. Donaldson Lithographing Company (1903) ${ }^{7}$ la Suprema Corte de los Estados Unidos dictaminó que: "La personalidad siempre contiene algo único. Esta expresa su singularidad incluso en la escritura, y un grado modesto de arte tiene [siempre] algo irreductible, lo que es [una cualidad] única del hombre". De hecho, la consagración de los derechos morales del autor viene a ser una consecuencia de la doctrina romántica del autor. Todavía en el siglo XXI, el significado de lo creativo sigue siendo debatido y controversial. La psicología pareciera ser categórica al considerar que la creatividad solo es posible gracias a la intervención humana. Como dice Monreal (citado en Velasco, 2007, p. 20) la creatividad es propia de la psique humana y, por ende, sin el ser humano la creatividad no existiría.

Este es el motivo por el que el sistema actual excluye la posibilidad de la autoría algorítmica, esto es que un algoritmo de inteligencia artificial pueda eventualmente considerarse como autor de las obras creativas que genera. Pero lo cierto es que el concepto de "creatividad" se entiende desde una concepción más amplia que el mero intelecto humano. Como indica Flanagan (citado por Strokes, 2005) indica:

La creatividad se muestra al dar existencia a algo novedoso. Lo esencial aquí está en la novedad y en la no existencia previa de la idea o producto. La creatividad es demostrada inventando o descubriendo una solución a un problema y en la demostración de cualidades excepcionales en la solución del mismo. (p. 10)

En ese sentido, la creatividad se asociaría a circunstancias más objetivas, medibles y cuantificables en contraposición a las concepciones netamente subjetivas de la creatividad.

Los sistemas de inteligencia artificial se describen por las siguientes características: son innovadores, autónomos, impredecibles, independientes, racionales, susceptibles de aprendizaje, eficientes, precisos e, incluso, algunos son capaces de tomar decisiones autónomas. El debate se centra en si todas estas características son suficientes para que una obra de arte generada artificialmente pueda gozar de originalidad conforme al derecho de autor (Shlomit et al., 2009).

En principio, al menos desde el punto de vista teórico, una obra generada por computadora podría eventualmente considerarse como original con base en los criterios de originalidad objetiva, la que se asocia a la figura de la novedad. Esto

7 Suprema Corte de los Estados Unidos, Bleistein v. Donaldson Lithographing Co., 188 U.S. 239 (1903). 
no deja de significar un reto para el sistema actual de la propiedad intelectual. Como dice Boden (2004, p. 14), la creatividad es una característica fundamental de la inteligencia humana, por lo que es un reto inexorable para la inteligencia artificial. Pero lo cierto es que, de una manera progresiva, los programas informáticos cada vez son más capaces para realizar tareas que requieren altos grados de expresión creativa. De manera que si una obra generada por inteligencia artificial es lo suficientemente creativa, podría considerarse protegida por el derecho de autor, debiendo determinarse su autoría.

Debe indicarse que no se trata de que existan obras más creativas que otras, sino que no toda creación intelectual tiene el grado de originalidad suficiente para alcanzar protección autoral.

Debe aclararse que el presente artículo no pretende crear criterios de originalidad. Como señala Ruipérez de Azcárate (2012), la originalidad es una condición sine qua non para alcanzar la protección autoral y es el requisito que adquiere mayor relevancia para valorar la susceptibilidad de protección de una creación, siendo la originalidad la que traza la línea divisoria entre las meras creaciones (no protegidas) y las obras efectivamente protegidas por el derecho de autor. Por lo que la exigencia y la valoración de la originalidad de una creación o producto creativo son indispensables para la determinación de la protección de una obra. De manera que para determinar la autoría de las obras generadas por inteligencia artificial es imprescindible precisar el grado de influencia del programa informático en el proceso creativo. En el caso de las creaciones generadas por inteligencia artificial, si se aplicara el criterio de originalidad objetiva, los parámetros asociados a la determinación de la altura creativa de los productos generados por computadora tendrían una relación directa, a su vez, con la determinación de la autoría de la obra algorítmica.

Ahora bien, ¿cómo puede determinarse si una obra generada por computadora es lo suficientemente creativa para alcanzar protección autoral? Esto suele ser una tarea compleja y, para ello, comúnmente se emplean diferentes versiones del mencionado Test de Turing para determinar si la obra está dotada de creatividad y originalidad. En el caso de que una o varias personas no puedan discriminar si una determinada obra fue producida por una computadora o por un ser humano, entonces esto significaría que la obra ha pasado el test.

De esa forma han surgido diferentes versiones informales del Test de Turing para evaluar la originalidad y creatividad de las obras. Tal es el caso de Zackary Scholl, estudiante de la Universidad de Duke que en el año 2010 empezó a enviar escrituras de poesía generadas por software a sitios web donde habitualmente las personas publican sus estrofas con el propósito de medir la reacción 
de las personas, sin que estas supieran que esos poemas habían sido generados por inteligencia artificial. La retroalimentación de los poemas fue abrumadoramente positiva. En el año 2011, Scholl decidió enviar los poemas generados por el algoritmo a diversas revistas especializadas en literatura, siendo en principio rechazados hasta que uno de ellos fue aceptado por The Duke Literary Journal (la revista literaria de la prestigiosa Universidad de Duke). ${ }^{8}$

Cuatro años más tarde, Scholl -siendo doctorando en Biología Computacional- sostuvo que aun cuando solo uno de los poemas generados por el algoritmo había sido publicado, este habría pasado el test de originalidad al ser aceptado en una revista de prestigio sin que se detectara que la obra era producto de un sistema artificial.

Otro ejemplo similar es el caso de BotPoet, que es un sitio web que sirve como plataforma para comparar poesía generada por humanos y poesía generada por computadoras. Esta plataforma le muestra los dos poemas al usuario, quien deberá efectuar una votación e indicar cuál de ellos considera que fue creado por el ser humano y cuál fue creado por inteligencia artificial (Kurt, 2018, p. 12).

Por su parte, un grupo de investigadores de Inteligencia Computacional de la Universidad de Málaga (España) ha desarrollado un software llamado Iamus, que es capaz de componer música clásica. El equipo liderado por el profesor Francisco Vico, del área de Ciencias Computacionales, utiliza técnicas biomiméticas para aplicar principios de la genética y de la teoría darwiniana de la evolución a los lenguajes informáticos, con el propósito de crear nuevos modelos de inteligencia artificial.

La música generada por el algoritmo de su laboratorio ha sido interpretada por diferentes agrupaciones musicales, incluyendo la Orquesta Sinfónica de Londres, habiendo pasado desapercibida por las personas sin detectar que se trataba de creaciones generadas por un programa informático. ${ }^{9}$

Pero no todos confían en este tipo de pruebas. Algunos consideran que las versiones informales del test de originalidad se han utilizado de manera indiscriminada y poco confiable. Opinan que al realizar las evaluaciones fuera de un contexto determinado, trae como consecuencia la falta de confiabilidad de dichas pruebas.

Esto, aunado al hecho de que el auténtico Test de Turing todavía no ha sido superado por ningún sistema inteligente, mal podría entonces (opinan algunos) validarse su efectividad en relación con la altura creativa de una obra. Además, no

8 Ver: https://www.vice.com/en_us/article/vvbxxd/the-poem-that-passed-the-turing-test.

9 Ver: https:/www.europapress.es/ciencia/noticia-cientificos-universidad-malaga-desarrolla-ordenador-capaz-componer-musica-clasica-20120701185848.html. 
puede esperarse que los sistemas artificiales posean el mismo nivel de la creatividad humana, lo que no necesariamente significa que estas carezcan de creatividad (Jordanous, 2012, pp. 246-279).

Otra crítica que se le hace a las variaciones del test de originalidad recae en la naturaleza del dominio de la obra y del grado de altura creativa que se le exige en consecuencia. Hay obras que siempre requieren del mismo nivel de altura creativa, como los chistes o las composiciones musicales, por ejemplo, pero hay otras cuyo grado de expresión creativa y originalidad es más complejo de determinar porque este varía no solo en función del resultado final de la obra, sino también del proceso creativo, como es el caso de las obras de arte visuales (López de Mántaras Badia, 2013).

Ahora bien, ¿dónde recae la altura creativa de una obra, en el proceso o en el resultado? La creatividad de un ser humano se manifiesta en los artefactos que produce, es decir, únicamente en el resultado, pudiendo ignorarse el proceso para su producción. Por tales motivos, lo mismo se debe aplicar para los sistemas creativos artificiales. Aunado a ello, el proceso subyacente no siempre es observable, por lo que para Ritchie basta con analizar el resultado para determinar la originalidad de la obra (Ritchie y Hanna, 1984).

Otros doctrinarios, en cambio, consideran que lo indispensable para determinar la originalidad de productos generados por inteligencia artificial recae en el proceso de la creación y no en su resultado. Esto se debe a que la impronta inteligente del algoritmo no sería equiparable al esfuerzo creativo del ser humano. Debido a ello, se debe poner el acento en el proceso creativo y determinar el nivel de procesamiento y cómputo del sistema (Navas, 2018, p. 13).

En ese sentido, Colton (2008) fue uno de los pioneros en sugerir que la creatividad debe ser medida tanto en el proceso como en el producto final. En ese orden de ideas, el primer factor que debería tomarse en cuenta para evaluar la creatividad de un sistema es el resultado y, posteriormente, analizar el proceso creativo que desarrolla el software para finalmente evaluar su funcionalidad. Entonces, según este criterio, ambos elementos deben analizarse para determinar si una obra creada por un algoritmo es o no lo suficientemente creativa.

Más allá del debate sobre si la altura creativa versa en el proceso o en el resultado, para la investigadora del Kings College de Londres, Anna Jourdanous (2012, p. 6), la evaluación no debe centrarse categóricamente ni en el uno ni en el otro, sino que todo dependerá del sistema inteligente en cuestión, del producto a analizar y de las características específicas de cada caso concreto. En ese sentido, propone un método para evaluar la creatividad de los sistemas inteligentes, que consta de tres pasos. 
En primer lugar, debe identificarse y fijarse por el evaluador un criterio específico de creatividad. La definición referida debe darse tanto en un contexto genérico como en un contexto específico, dependiendo del dominio artístico del que se trate (poesía, música y arte visual, entre otros). En segundo lugar, se deben establecer parámetros concretos para evaluar la creatividad del sistema. Finalmente, debe valorarse si el sistema cumple o no con los parámetros previamente establecidos. Esta valoración podrá ser de índole cualitativa o cuantitativa, dependiendo del caso particular.

Esto denota la relevancia de establecer criterios claros de originalidad y altura creativa en relación con las obras generadas por inteligencia artificial. Cabe destacar que, con respecto a la creatividad computacional, en la mayoría de los casos la novedad y la utilidad se consideran como criterios esenciales para efectuar el análisis de creatividad. Esto coincide con el criterio de originalidad objetiva y la definición aportada por Boden (2004) que implica la creación de algo novedoso por medio de la solución de un problema. De manera que en lugar de considerar la originalidad como un reflejo de la personalidad del autor (criterio subjetivo), en el caso de los productos creativos generados por los algoritmos resulta más adecuado aplicar los criterios de originalidad objetiva.

Ahora bien, suponiendo que un producto creativo generado por inteligencia artificial alcance la suficiente altura creativa para considerarse protegido por el derecho de autor, ¿puede un sistema de inteligencia artificial considerarse titular originario de una obra algorítmica? La autoría algorítmica consiste en la cualidad de considerar como autor a un algoritmo o sistema de inteligencia artificial en relación con las obras originales creadas por este. Actualmente, uno de los aspectos más controvertidos por la doctrina es el debate sobre si se le debe reconocer o no autoría al algoritmo. La doctrina de la originalidad entendida como un manifiesto práctico de autoría no imposibilita la autoría algorítmica, es decir, la cualidad por medio de la cual un algoritmo se considera autor de las obras originales que ha creado de manera autónoma. Pero esto no deja de ser un aspecto sumamente controvertido, aunque su discusión no es reciente.

En el año 1974, el Congreso de los Estados Unidos creó la Comisión Nacional para Nuevos Usos Tecnológicos de Obras Protegidas por Derechos de Autor (CONTU, por sus siglas en inglés), que en un informe señaló lo siguiente:

La Comisión cree que no hay una base razonable para considerar que una computadora pueda contribuir como autor a una obra producida para su uso. La computadora como una cámara o una máquina de escribir, es un instrumento inerte, capaz de funcionar solo cuando es activado de manera directa o indirecta por un humano. (Denicola, 2016, p. 266) 
Por lo que en principio se entiende a las máquinas como un mero accesorio del ser humano durante el proceso creativo. Pero Arthur Miller, quien para ese entonces era uno de los Comisionados del Informe CONTU y actualmente es profesor de la escuela de leyes de la Universidad de Nueva York, no excluyó la posibilidad de otorgarle autoría al software, expresando que:

\section{Si llega el día en el que una computadora es realmente la única autora de una obra original artística, musical o literaria (sea una novela o un programa de computado- ra) el derecho de autor abrazará y Española lo suficientemente maleable para asim- ilar el desarrollo de obras generadas por computadoras. (Denicola, 2016, p. 268)}

En ese orden de ideas, Miller consideraba que no es descabellado pensar que eventualmente pueda otorgarse a una computadora la autoría de una obra algorítmica. En ese sentido, en el año 1986, la Oficina para Asistencia Tecnológica del Congreso de los Estados Unidos (OTA, por sus siglas en ingles) desarrolló un reporte para evaluar las implicaciones de las políticas de propiedad intelectual en los avances de redes de computadoras. A diferencia de la CONTU, la OTA sugiere que los programas de computación interactiva pueden legítimamente considerarse coautores del resultado que estos producen. ${ }^{10}$

De manera que, desde el punto de vista teórico, la autoría algorítmica es admisible en aquellos países donde no se excluye dicha posibilidad de manera expresa. Desde el punto de vista utilitario, es coherente otorgarle protección autoral a las obras generadas por computadora, porque esto generaría un mayor progreso económico y tecnológico a nivel social. Pero lo cierto es que hay diferentes criterios y posiciones doctrinales sobre cómo debe abordarse la cuestión de la autoría en relación con este tipo de obras. Por otra parte, Pamela Samuelson (1985) sostiene que las computadoras no han sido y no deberían tratarse como humanos, porque estas no tienen incentivo para crear, aunque sí es posible considerar a la máquina como "autor material", mientras que el autor -en sentido jurídicosería la persona física o jurídica que encargó la obra.

Para Annmarie Bridy (2012, p. 16), el sistema actual de derecho de autor no puede otorgar derechos de propiedad intelectual a un ente que no tiene personalidad jurídica y propone la doctrina del work made for hire como una solución al problema de la autoría algorítmica, considerándose como autor al empleador o a la persona que encarga la obra. De manera que tratar al programador como si fuera empleador solucionaría la cuestión de considerar otorgarle derechos a una máquina o algoritmo que carece de personalidad jurídica.

10 Ver: http://eric.ed.gov/PDFS/ED160122.pdf. 
Dicha doctrina se corresponde con la solución adoptada por el artículo 9 de la Ley de Derecho de Autor, Diseños y Patentes del Reino Unido. ${ }^{11}$ En lugar del autor como persona natural, o de otorgarle por el momento autoría al algoritmo, la autoría vendría a estar conformada por una ficción legal conformada por la empresa (persona natural o jurídica) que encarga la realización de la obra. Pero esta doctrina, como se ha mencionado anteriormente, da lugar a diferentes interpretaciones. Por su parte, Yanisky (2017) también se inclina por esta tesis, pero sostiene que los sistemas de inteligencia artificial deben considerarse como empleados del usuario y, por su parte, el usuario actuaría como empleador del sistema (Shlomit et al., 2009).

De esta manera, los algoritmos deben entenderse como un substituto del ser humano, pero no como un mero instrumento, sino como un empleado por este para generar las obras (ficción legal). Pero algunos autores consideran que los algoritmos sí pueden eventualmente llegar a considerarse como autores de las obras algorítmicas. En ese sentido, Andrew Wu sostiene que la inteligencia artificial puede ostentar autoría solo si cumple con ciertos requisitos: si produce obras que son originales, debe operar de manera autónoma y poseer la discreción sobre si producir o no la obra (Sorjamaa, 2016).

De esa forma, la autoría algorítmica estaría determinada por el grado de autonomía y de participación del algoritmo en el proceso creativo. Sin embargo, lo cierto es que el derecho (por el momento) no otorga personalidad jurídica a los sistemas de inteligencia artificial, lo que impide una eventual autoría algorítmica, al menos conforme al sistema actual del derecho de autor. Pero ante la exclusión de la autoría algorítmica ¿quién debe asumir la titularidad originaria de los derechos de autor sobre las obras generadas por sistemas de inteligencia artificial?

\section{Autoría de las obras generadas por inteligencia artificial en el sistema actual}

La legislación del Reino Unido es una de las pocas legislaciones que hacen referencia a las obras generadas por computadora y a la determinación de su autoría. El artículo 9 de la Ley de Derecho de Autor, Diseños y Patentes del Reino Unido es una de las primeras legislaciones que se refirió expresamente a la autoría sobre la obra algorítmica, prescribiendo lo siguiente: "En el caso de una obra literaria, dramática, musical o artística generada por computadora, se considerará que el autor es la persona que realiza los arreglos necesarios para la creación de la obra”.

11 Copyright, Designs and Patents Act, 1988, c. 48, \$ 178 (U.K.). 
Dicha norma ha sido fuertemente criticada porque no establece una posición clara y precisa en lo referente a la determinación de la autoría. Además, tampoco diferencia entre las obras algorítmicas y las obras producto de inteligencia artificial con intervención humana, lo que constituyen dos supuestos diferentes. Aunque en ambos casos las obras son generadas por software, la obra algorítmica es aquella en la que no existe una intervención humana durante el momento de la creación, es decir, el nivel de autonomía del algoritmo es considerablemente elevado.

Siguiendo con el análisis de la mencionada ley, también se le critica que excluye la protección de los derechos morales del autor. El artículo 78, que establece el derecho a ser identificado como autor de la obra, no sería aplicable a las obras generadas por computadora. De igual manera, el artículo 81 también excluye la aplicación del derecho a oponerse al tratamiento despectivo de la obra ${ }^{12}$ (derecho de integridad).

Aunque dicha legislación ha sido fuertemente criticada por la doctrina por no contemplar una solución práctica para la determinación de la autoría, sí debe señalarse que es una legislación pionera sobre la materia, atreviéndose a regular sobre aspectos que no son ni siquiera mencionados en casi ninguna de las legislaciones del mundo.

Ahora bien, ante la falta de una solución legal, surge de nuevo el interrogante: ¿quién debe considerarse como autor de las obras generadas por computadora? Este es un problema complejo para el derecho de autor y la dificultad radica en la determinación del grado de participación durante el proceso creativo. Además de ello, no existe una única teoría que aborde dicho problema y, como se ha comentado, son muy pocas las legislaciones que consagran una disposición expresa.

La cuestión fue abordada por las recomendaciones de la Organización Mundial de la Propiedad Intelectual (OMPI) y la UNESCO en un informe elaborado en 1982, en el que se recomienda conceder la autoría a aquella persona que, sin sus esfuerzos creativos, no hubiera podido crearse la obra. De manera general, el informe recomienda otorgarle la titularidad originaria al usuario del programa, pero también considera que el programador podría eventualmente considerarse como autor solo si hace contribuciones creativas. La Unión Europea, por su parte, trató el tema en 1998 en el Green Paper sobre Copyright. La comisión se inclina por concederle la titularidad al usuario cuando el programa es esencialmente una mera herramienta en el proceso creativo (Sorjamaa, 2016).

Pero una de las características de los sistemas creativos artificiales es que pueden generar obras al combinar data de diferentes fuentes: por parte de las ins-

12 Copyright, Designs and Patents Act, 1988, c. 48, § 178 (U.K.). 
trucciones indicadas por el programador, por parte del material suministrado por el usuario del programa y también de la información obtenida por medio de las operaciones propias del programa. Las técnicas de deep learning y la minería de datos hacen aún más difícil la determinación de la autoría en este tipo de obras.

Excluyéndose, por el momento, la autoría algorítmica, el primer sujeto en el que podría eventualmente recaer la titularidad originaria es en el programador. Si la obra algorítmica se sustenta en el código del programa, entonces ¿̨o se justificaría que los derechos de autor le correspondan al autor del software? Este es el principal argumento a favor del programador. Debido a que el programador es el creador del algoritmo y este, a su vez, es el resultado de su esfuerzo creativo, una obra generada por computadora no existiría nunca sin el programador del algoritmo.

De esta manera, algunos argumentan que la obra generada por inteligencia artificial debe entenderse como una obra derivada del programa de ordenador, pero todo esto dependerá de las especificidades de cada obra en particular. Solo en el caso de que la obra posea partes sustanciales del código del programa, entonces el programador podría eventualmente reclamar los respectivos derechos en relación con la producción de una obra derivada (Perry y Margoni, 2010)

En principio, otorgarle derechos de autor al programador incentivaría la creación de sistemas de creatividad artificial, lo que fomentaría la creatividad y la innovación generando grandes beneficios para la sociedad. Pero el hecho de considerar al programador como autor también ha recibido fuertes críticas por parte de la doctrina, principalmente porque este no crea de manera directa e intencional la obra generada por inteligencia artificial. En muchos casos, es el propio software y no el programador el que determina la forma y el resultado final de la obra, siendo esa forma de expresión la que vendría a estar protegida por el derecho de autor (Sorjamaa, 2016). Otro argumento en contra es que el programador ya se considera autor del software informático, ostentando sus respectivos derechos morales y patrimoniales, lo que hace redundante e innecesario otorgarle además los derechos sobre las obras que el programa genere (Glasser, 2001).

El segundo sujeto que se perfila como potencial autor es el usuario del programa. El usuario es la persona que generalmente se asocia con una participación directa en el proceso creativo. Debe aclararse que no cualquier usuario eventualmente calificaría como autor de las obras generadas por computadora, solamente aquel cuya participación fuera significativa podría ostentar la titularidad originaria. Dicho aporte no se refiere a una mera actividad mecánica, como presionar un botón o hacer un simple clic (Perry y Margoni, 2010). Algunos consideran que, incluso, no bastaría con una contribución significativa, sino que su actuación debería ser indispensable durante el proceso creativo. Hay muchos argumentos para 
considerar al usuario como titular originario de los derechos de autor. De hecho, la Comisión Nacional para el uso de Nuevas Tecnologías sobre obras protegidas por derechos de autor del Congreso de los Estados Unidos, en su informe CONTU, determinó que es el usuario del programa quien debe considerarse como autor de las obras generadas por computadora. En el caso de que el algoritmo esté actuando como una mera herramienta, la titularidad originaria en principio debe recaer sobre el usuario. Es el usuario el que tiene la intención de crear las obras al proveer al programa con las instrucciones iniciales. Si bien el programador toma la decisión de crear el programa, es el usuario el que toma la decisión de crear las obras, pero en el caso de que su contribución sea mínima, entonces sería difícil el justificar su reconocimiento como autor (Sorjamaa, 2016).

Por su parte, Saiz García (2019) considera que otra alternativa es otorgarle un derecho sui generis a las obras algorítmicas, de manera similar a la protección que poseen las bases de datos conforme al derecho comunitario europeo. Sin embargo, esta podría no ser en principio la mejor opción, debido a los problemas para su aplicación práctica y a las particularidades relativas a las prestaciones propias del derecho de autor.

Respecto a la autoría algorítmica, Peter Patchen, decano de artes digitales del Pratt Institute en la ciudad de Nueva York, considera que hasta tanto el algoritmo no adquiera personalidad jurídica tampoco debe tener crédito en la participación autoral, pero sí considera que tanto el usuario como el programador merecen reconocimiento y la autoría debe ser compartida entre ambos sujetos (Kaminski, 2017).

En ese supuesto, se hablaría de una autoría conjunta, que aplicaría cuando las contribuciones de cada coautor constituyen una contribución independiente y existe una intención de ambas partes de ser coautores (Brown, 2017). En ese caso podría aplicarse un régimen similar al que se les atribuye a las obras colectivas y a las obras en colaboración, dependiendo del caso particular. Tal como sucede en relación con los programas de computadora, en el caso de las obras audiovisuales y en las obras anónimas existe la posibilidad de atribuir autoría tanto a una persona natural como a una persona jurídica de conformidad con el artículo 15 del Convenio de Berna.

No obstante, no toda contribución colaborativa necesariamente produce una obra en colaboración. Para ello, es necesario que cada una de las contribuciones represente una expresión original protegible por el derecho de autor. Aunado a esto, la tesis de la autoría conjunta también es criticada por razones de inequidad entre la participación directa del usuario en el proceso creativo y la participación indirecta del programador (Glasser, 2001). De manera que existen diferentes 
criterios para la determinación de la autoría de las obras generadas por computadora. Todas las propuestas poseen argumentos a favor y en contra, sin que exista un criterio uniforme para la determinación de la autoría.

Nina Brown plantea el siguiente caso hipotético: el supuesto de una aplicación sencilla que genera artículos y notas periodísticas (similar al sistema Dreamwriter, cuyos artículos son reconocidos en China como obras protegidas de conformidad con la decisión del Tribunal de Shenzhen). El software que plantea el caso hipotético es vendido a una organización para la creación de nuevas historias. El grado de participación de la organización en la generación de las obras es bastante relativo. Puede que el encargado de la empresa solo se limite a encender el algoritmo, en cuyo caso, la empresa no tendría derechos de autor sobre la obra (Brown, 2017). En este caso, ¿a quién le debe corresponder la autoría? ¿Le debe corresponder al programador, a la compañía o a ambos? Por lo que la determinación de la autoría no es tan sencilla para este caso hipotético.

En otros casos la solución no es tan compleja (aparentemente). Tal es el caso de las obras generadas por AARON (Kurt, 2018), un programa informático que fue creado en 1973 por Harold Cohen, un reconocido artista y docente británico cuyo propósito era generar obras de arte de manera automática. Cohen describió el proceso de creación de AARON similar al proceso de enseñar a un niño a dibujar, ${ }^{13}$ refiriéndose al programa como una entidad, como un pupilo que necesitaba aprender a dibujar. Cohen logró que AARON aprendiera a tomar decisiones autónomas sobre el trazado de las formas. Al principio, el sistema requería de un trabajo colaborativo, pero cuando Cohen falleció -en abril de 2016-, el software ya tenía la plena capacidad de manejarse de una manera autónoma en cuanto a la composición, la determinación del color y otros aspectos del trazado. El programa aprendió incluso a reconocer cuando una obra de arte ya había alcanzado su acabado final.

La titularidad originaria de dichas obras le correspondería a su creador, Harold Cohen (o a sus respectivos herederos y/o derechohabientes), debido a que él fue tanto el programador como el usuario del sistema, por lo que su participación en el proceso creativo es indiscutible y, por ende, le corresponde la autoría de las obras generadas por AARON. Sin embargo, también habría que precisar el grado de autonomía del sistema para determinar si se trata de una obra algorítmica, en cuyo caso, conforme a la legislación actual, lo más probable es que la obra pase a formar parte del dominio público por carecer de contribución humana.

13 Ver: https://www.nytimes.com/2016/05/07/arts/design/harold-cohen-a-pioneer-of-computer-generated-art-dies-at-87.html. 
La determinación de la autoría es mucho más difícil de realizar cuando la relación entre el programador y el usuario es estrecha y, por ende, se hace más complejo delimitar la participación individual de cada sujeto. En la medida en que la participación del programador es mayor, la participación del usuario tiende a disminuir y viceversa. Sin embargo, los casos más difíciles involucran diferentes grados de participación, lo que hace aún más difícil la determinación de la autoría (Boyden, 2016).

Un ejemplo es el caso del proyecto The Next Rembrandt, en el que participó un equipo conformado por una gran cantidad de investigadores para la realización de la obra final. Hace casi cuatro siglos, falleció el pintor neerlandés Rembrandt van Rijn, reconocido como uno de los grandes maestros de la pintura barroca y considerado el artista más importante de los Países Bajos. En el año 2016, se creó un grupo de investigación conformado por Microsoft, la Universidad Técnica de Delft y los museos Mauritshuis y Rembrandthuis, de los Países Bajos, con el propósito de lograr desarrollar por medio de sistemas informáticos y mecanismos de impresión 3D una nueva obra de arte con un estilo similar al del artista neerlandés, a cuyo retrato denominaron The Next Rembrandt (Brow, 2017).

Luego de almacenar la colección virtual, las computadoras lograron determinar ciertos patrones en los retratos de la colección que corresponden con el estilo del artista y, utilizando complejos métodos computacionales de deep learning (aprendizaje automático), lograron obtener como resultado una obra que, desde el punto de creativo, está dotado de elementos característicos y representativos de las creaciones desarrolladas por Rembrandt. Dicha obra de arte se caracteriza porque no fue creada por una persona física, sino que fue generada producto de la combinación de complejos y sofisticados mecanismos de inteligencia artificial.

En este caso, no queda claro si tanto los científicos de datos como los ingenieros, desarrolladores, consultores e historiadores que participaron en el proyecto pudieran reclamar una participación sobre los derechos de la obra o una eventual participación conjunta. Aunque también podría argumentarse que la autoría le corresponde a las organizaciones que encargaron la realización de la obra de conformidad con la doctrina work made for hire, en este caso, Microsoft, la Universidad Técnica de Delft y los museos Mauritshuis y Rembrandthuis, de los Países Bajos.

La inteligencia artificial también está cambiando la industria de la música. Gil Weinberg, director del Georgia Tech Center for Music Technology, está creando en su laboratorio nuevas expresiones de creatividad artificial. Entre ellas se destaca Shimon, un robot capaz de generar improvisaciones musicales. Una vez que el robot escucha las interpretaciones musicales desarrolladas por seres humanos, posee la habilidad de realizar improvisaciones a partir de estas. Lo que hace esta 
tecnología es reconocer patrones musicales por medio del deep learning, para luego hacer modificaciones y crear composiciones musicales nuevas y originales. ${ }^{14}$

Todo indica que los algoritmos tienen la capacidad para disrumpir la industria de la música y desafiar la actual legislación autoral. Un ejemplo de ello es la obra musical Daddy's Car, generada por el software Flow Machines, en el que el compositor, Benoit Carré, seleccionó un conjunto de partituras que servirían de inspiración para determinar el estilo de la obra. Pero si bien el compositor se encargó de instruir a la plataforma y de crear las letras, fue el sistema informático el que realizó las variaciones y las combinaciones necesarias para generar el resultado final. Esto evidencia una auténtica colaboración entre el ser humano y la inteligencia artificial para crear un álbum completo denominado Hello World, en el que tanto el sistema informático como Benoit realizaron importantes arreglos musicales para componer las canciones, cuyo resultado final cuenta con los elementos de originalidad para considerarse una obra protegida por el derecho de autor (Díaz, 2016).

Pero además de las melodías a las que estamos acostumbrados, los algoritmos también pueden crear novedosos sonidos, haciendo combinaciones de elementos preexistentes para dotar al resultado de novedad y originalidad. Recientemente, la reconocida cantante islandesa Björk ha colaborado con Microsoft IA y el hotel Sister City de Nueva York para crear música generativa capaz de cambiar su sonido de acuerdo al estado del clima. El algoritmo de inteligencia artificial obtiene los datos climatológicos y, dependiendo de los eventos del clima, genera la música y hace los arreglos musicales. El producto final estaría conformado por sonidos nuevos que constituyen nuevas formas de creatividad artificial que difícilmente podrían llegar ser interpretados por seres humanos. ${ }^{15}$

En estos casos -tanto en el de las obras generadas por Flow Machines como las generadas en el proyecto de Microsoft I-, en relación con la determinación de la autoría, podría plantearse una eventual autoría conjunta, pero al no existir una disposición legal expresa, la determinación de la autoría es debatible y genera un vacío legislativo. En principio, puede sostenerse que el usuario del programa -en el primer caso, el compositor Benoit- es quien debería ostentar la cualidad de autor de la obra, ya que efectuó los arreglos necesarios para su composición musical. Sin embargo, al tratarse de una obra por encargo, también podría argumentarse que, conforme a la doctrina work made for hire, los derechos de autor le corresponden como ficción legal a las compañías desarrolladoras, como serían Sony CSL o Microsoft IA para los casos anteriormente descritos.

14 Ver: https://gtcmt.gatech.edu/.

15 Ver: https://www.microsoft.com/inculture/musicxtech/bjork/. 
Esto demuestra que la determinación del grado de participación y la autoría en el proceso creativo de una obra generada por computadora es un proceso complejo y actualmente sin una solución legal concreta, aunado a la existencia de diferentes criterios doctrinales que hacen aún más intrincada la determinación de la autoría en este tipo de obras.

Si bien el creador de un software ostenta los derechos de autor sobre este, no está claro a quién le corresponde la titularidad originaria de las obras generadas por el algoritmo cuando este ha actuado de manera autónoma al tomar decisiones individuales y de manera independiente. Pero respecto a las obras algorítmicas, parece haber cierto consenso doctrinal. Debido a que carecen de una intervención humana significativa, conforme a la legislación actual quedarían a beneficio del dominio público al no haber un autor humano al que le corresponda la titularidad originaria. Sin embargo, esto trae como consecuencia una falta de incentivo para estimular el desarrollo de nuevas obras creativas, lo que no es beneficioso para la sociedad ni para el desarrollo cultural (Perry y Margoni, 2010).

Pero como indica Azuaje Pirela (2020), el hecho de que no sea posible designar como autor a una máquina no implica necesariamente que los productos de la IA, en todos los casos, estarán en el dominio público De manera que en el caso de las obras generadas por inteligencia artificial con intervención humana, la autoría le correspondería en principio a la persona natural que hubiere participado en el proceso creativo de manera directa.

Sin embargo, ante la necesidad de despejar los vacíos legislativos respecto a estos temas, el Parlamento Europeo, ${ }^{16}$ en su reporte del ańo 2017 dedicado al impacto de la inteligencia artificial en relación con los estándares del derecho civil, considera que es necesario actualizar la legislación en esta materia ante la innegable sofisticación de los sistemas informáticos actuales, cuyo grado de autonomía se incrementa significativamente debido a los constantes avances en esta tecnología.

Por su parte, la Organización Mundial de la Propiedad Intelectual inició desde el año 2019 un proceso de consulta pública sobre los tópicos emergentes respecto a la inteligencia artificial y nuevas políticas en materia de propiedad intelectual. En esa consulta se invita tanto a académicos, representantes de Estado y a los diferentes actores que hacen vida en el mundo de la propiedad intelectual a formular propuestas y debatir sobre eventuales soluciones normativas con respecto a los dilemas que plantean los avances en materia de inteligencia artificial en relación con la propiedad intelectual.

Recientemente, la Comisión de Asuntos Jurídicos del Parlamento Europeo

16 Ver: http://www.europarl.europa.eu/doceo/document/A-8-2017-0005_ES.html?redirect. 
publicó un informe referente a los derechos de propiedad intelectual para el desarrollo de las tecnologías relativas a la inteligencia artificial. En este informe, entre diversas cuestiones, recomienda la evaluación sectorial de las implicaciones de la inteligencia artificial en relación con la propiedad intelectual, indicando especialmente la relevancia que tiene la determinación del grado de intervención humana, la autonomía del algoritmo y la importancia del origen de los datos y del material protegido por el derecho de autor. El informe considera necesario profundizar en el estudio de la valoración del origen humano de los datos en los algoritmos de inteligencia artificial. ${ }^{17} \mathrm{El}$ mencionado informe sugiere que la automatización del proceso de generación de contenidos creativos por parte de sistemas expertos de inteligencia artificial plantea importantes cuestiones relativas a la autoría y titularidad de los derechos de propiedad intelectual, y considera que no sería adecuado tratar de dotar a los algoritmos de IA de personalidad jurídica, al poner de relieve el impacto negativo que generaría dicha posibilidad en los incentivos para los creadores humanos. El informe también señala la diferencia entre las creaciones humanas asistidas por la IA y las creaciones generadas por la IA, estas últimas plantean importantes retos para la protección autoral y sostiene que en los casos en los que la IA solo se usa como herramienta para ayudar al autor en el proceso de creación, las normas actuales de propiedad intelectual actual siguen siendo aplicables. También se indica la relevancia de la protección autoral de las obras generadas por IA, esto como mecanismo para fomentar las inversiones, el desarrollo tecnológico. Además, sugiere debatir políticas adecuadas en este ámbito conforme a la estrategia de materialización del Mercado Único Digital de Europa.

Lo cierto es que actualmente el sistema no da respuesta a los diferentes escenarios que plantean los avances de estas nuevas tecnologías y deben procurarse soluciones normativas que cuenten con un consenso en la materia para lograr una armonización de criterios. Estas reformas legislativas deben contemplar un balance entre los intereses de todos los involucrados en el proceso creativo, incluyendo a los programadores, usuarios, inversionistas y los intereses del público en general, con el propósito de incentivar la creatividad y generar nuevas formas de expresión artística acorde a los retos tecnológicos actuales, tomando como premisa el fomento y la protección de la creatividad y la innovación. Esto es esencial para el futuro desarrollo de la industria de la inteligencia artificial y para garantizar el fomento de una adecuada protección a la propiedad intelectual.

17 Ver: https://www.europarl.europa.eu/doceo/document/A-9-2020-0176_ES.pdf. 


\section{Conclusiones}

La cualidad de autor, salvo en los casos de las ficciones jurídicas, por el momento solo recae en el ser humano, excluyéndose la posibilidad de otorgarle autoría a los sistemas de inteligencia artificial. Actualmente, se debate si la autoría de este tipo de obras debe recaer en el programador, en el usuario del sistema o en la persona que encarga la obra. También la doctrina plantea otras soluciones, como la work made for hire, la autoría conjunta e incluso la posibilidad de consagrar un nuevo derecho sui generis. Pero lo cierto es que no existe un criterio uniforme y debe procurarse una regulación acorde a los nuevos desarrollos tecnológicos y con base en el consenso y la cooperación de los diferentes actores que hacen vida en el mundo de la propiedad intelectual.

\section{Bibliografía}

Antequera Parilli, R y Gómez, G. (1999). Legislación Sobre Derechos de Autor y Derechos Conexos. Caracas: Editorial Jurídica Venezolana.

Azuaje Pirela, M. (2020). Protección jurídica de los productos de la inteligencia artificial en el sistema de propiedad intelectual. Revista Jurídica Austral, 1(1), 319-342. https://doi. org/10.26422/RJA.2020.0101.azu.

Bercovitz, R. (2007). Comentarios al art. 10 LPI. En Bercovitz, R. (Coord.), Comentarios a la Ley de Propiedad Intelectual ( $3^{\mathrm{a}}$ ed., pp. 151 y ss.). Madrid: Tecnos.

Boden, M. (2004). Creativity and Artificial Intelligence. Artificial Intelligence, 103(1), 347-356.

Boyden, B. (2016). Emergent Works. Columbia Journal of Law \& The Arts, 39(3), pp. 377-394). https://journals.cdrs.columbia.edu/wp-content/uploads/sites/14/2016/06/7-39.3-Boyden.pdf.

Brandt, A. K. y Eagleman, D. (2017). The runaway species: How human creativity remakes the world. Edimburgo: Canongate Books.

Bridy, A. (2012). Coding Creativity: Copyright and the Artificially Intelligent Author. Stanford Technology Law Review, 5, 1-28. https://ssrn.com/abstract=1888622.

Brow, N. I. (2017). Artificial Authors: a Case For Copyright in Computer-Generated Works. Science and Technology Law Review, 20(1), 1-41. https://doi.org/10.7916/stlr.v20i1.4766.

Colton, S. (2008). Creativity vs the perception of creativity in computational systems. https://www. semanticscholar.org/paper/Creativity-Versus-the-Perception-of-Creativity-in-Colton/a2eab$9219192 \mathrm{~b} 14 f 5019 \mathrm{e} 346566668$ bf4986f591.

Denicola, R. (2016). Ex Machina: Copyright Protection for Computer-Generated Works. Rutgers University Law Review, 251. https://ssrn.com/abstract=3007842.

Díaz, J. (2016). Daddy's Car: inteligencia artificial como herramienta facilitadora de los derechos de autor. Revista de la Propiedad Inmaterial, (22), 83-100.

Fernández, H. (2011). Manual de los Derechos de Autor. Buenos Aires: Editorial Heliasta. 
Frankish, K. y Ramsey, W. (Eds.). (2014). The Cambridge Handbook of Artificial Intelligence. Cambridge: Cambridge University Press. https://doi.org/10.1017/CBO9781139046855.

García, T. (2016). Análisis del criterio de originalidad para la tutela de la obra en el contexto de la ley de propiedad intelectual. Anuario Jurídico y Económico Escurialense, XLIX, 251-274.

Ginsburg, J. C. (2003). The Concept of Authorship in Comparative Copyright Law. Columbia Law School. https://ssrn.com/abstract=368481.

Ginsburg, J. C. (2018). People not machines: Authorship and what it means in Bern Convention. The International Review of Intellectual Property and Competition Law, 49(2), 131-135. https://link.springer.com/article/10.1007/s40319-018-0670-x.

Glasser, D. (2001). Copyrights in Computer-Generated Works: Whom, if Anyone, Do We Reward? Duke Law \& Technology Review, 1. https://scholarship.law.duke.edu/dltr/vol1/ iss $1 / 24 /$.

Gopinath R., Ajay R. y Sanjay C. (2019). An Introduction to Machine Learning. California: Springer. https://www.springer.com/gp/book/9783030157289.

Grimmelmann, J. (2016). Copyright for Literate Robots. Iowa Law Review, (101). 657-681.

Hertzmamm, A. (2018). Can Computers Create Art? https://arxiv.org/pdf/1801.04486.pdf.

Hobbes, T. (1989). Leviatán, la Materia forma y poder de un estado eclesiástico y civil (1ª ed.). Madrid: Alianza.

Hristov, K. (2017). Artificial intelligence and the copyright dilemma. IDEA: The IP Law Review, 57(431). https://papers.ssrn.com/sol3/papers.cfm?abstract_id=2976428.

Jordanous, A. (2012). A Standardised Procedure for Evaluating Creative Systems: Computational Creativity Evaluation Based on What it is to be Creative. Cognitive Computation, 4(3). https://doi.org/10.1007/s12559-012-9156-1.

Kaminski. M. (2017). Authorship, Disrupted: AI Authors in Copyright and First Amendment Law. Uniersity of California Davis Law Review, 51, pp. 589-616. https://ssrn.com/abstract $=3086912$.

Kulstad, M. y Laurence, C. (2013). Leibniz's Philosophy of Mind. The Stanford Encyclopedia of Philosophy. https://plato.stanford.edu/cgi-bin/encyclopedia/archinfo.cgi?entry=leibniz-mind.

Kurt, D. (2018). Artistic Creativity in Artificial Intelligence. https://theses.ubn.ru.nl/bitstream/ handle/123456789/5631/Kurt\%2C_D.E._1.pdf?sequence=1.

Lipszyc, D. (2017). Derecho de autor y derechos conexos. Bogotá: Centro Regional para el Fomento del Libro en América Latina y el Caribe (CERLALC). https:/cerlalc.org/publicaciones/ derecho-de-autor-y-derechos-conexos/.

López de Mántaras Badia, R. (2013). Creatividad computacional. Arbor, 189(764), a082. http:// dx.doi.org/10.3989/arbor.2013.764n6005.

Navas, S. (2018). Obras generadas por algoritmos. En torno a su posible protección jurídica. Revista de Derecho Civil, 5(2), pp. 273-291.

Parlamento Europeo. (2017). Informe con recomendaciones destinadas a la Comisión sobre normas de Derecho Civil sobre Robótica. A8-0005/2017. http://www.europarl.europa.eu/doceo/document/A-8-2017-0005_ES.pdf.

Pease, A. y Colton, S. (2011). On impact and evaluation in computational creativity: a discussion of the Turing Test and an alternative proposal. En Kazakov, D. y Tsoulas, G. (Eds.), Proceedings of AISB '11: computing and philosophy (pp. 15-22). York: Society for the Study of Artificial Intelligence and Simulation of Behaviour. 
Perry, M. y Margoni, T. (2010). From Music Tracks to Google Maps: Who Owns Computer Generated Works? Computer Law and Security Review, 26, 621-629. https://ssrn.com/abstract $=1647584$.

Ramalho, A. (2017). Will Robots Rule the (Artistic) World? A Proposed Model for the Legal Status of Creations by Artificial Intelligence Systems. https://ssrn.com/abstract=2987757.

Real Academia Española (s.f.). Diccionario de la lengua española (23a ed., versión 23.3 en línea). https://dle.rae.es/inteligencia.

Ríos Ruiz, W. (2001). Los sistemas de inteligencia artificial y la propiedad intelectual de las obras creadas, producidas o generadas mediante ordenador. Revista La Propiedad Inmaterial, (3), 5-14.

Ritchie, G. D. y Hanna, F. K. (1984). AM: A case study in AI methodology. Artificial Intelligence, (23), 249-268.

Ruipérez de Azcárate, C. (2012). Las Obras del Espíritu y su Originalidad. Madrid: Editorial Reus.

Saiz García, C. (2019). Las obras creadas por sistemas de inteligencia artificial y su protección por el derecho de autor. InDret, 1. https://papers.ssrn.com/sol3/papers.cfm?abstract_ id $=3365458$.

Samuelson, P. (1985). Allocating Ownership rights in computer-generated works. University of Pittsburgh Law Review, 47. https:/www.semanticscholar.org/paper/Allocating-Ownership-Rights-in-Computer-Generated-Samuelson/accb9aafa77f06f97b2255bf3bdf836046c53714.

Shlomit Yanisky-Ravid y Vélez-Hernández, L. (2018). Copyrightability of Artworks Produced by Creative Robots and Originality: The Formality-Objective Model. Minnesota Journal of Law, Science \& Technology, 19(1), 1-53. https://scholarship.law.umn.edu/mjlst/vol19/iss1/11.

Sorjamaa. T. (2016). I, Author, autorship and copyright in the age of artificial intelligence. https:// helda.helsinki.fi/bitstream/handle/10138/166456/sorjamaa.pdf?sequence=3\&isAllowed=y.

Strokes, P. (2005). Creativity from Constraints: The Psychology of Breakthrough. Springer.

Velasco, P. (2007). Psicología y Creatividad. Una Revisión desde los autoretratos. Caracas: Fondo Editorial de humanidades de la Universidad Central de Venezuela. 
